\title{
Precisão, sensibilidade e confiança na percepção háptica de peso na presença ou ausência de movimento e visão
}

\author{
Márcio Rogério Penha \\ Universidade de São Paulo (Ribeirão Preto) \\ Ricardo Basso Garcia \\ Universidade Federal de Uberlândia \\ Igor Otto Douchkin \\ José Aparecido da Silva \\ Universidade de São Paulo (Ribeirão Preto)
}

\begin{abstract}
Resumo
Esta pesquisa investigou a influência do movimento muscular e da visão na precisão, sensibilidade e nível de confiança na percepção háptica de peso. Os participantes compararam pesos colocados sobre as palmas das mãos e, conforme a condição experimental, podiam movimentar ou não os braços, bem como visualizar ou não suas mãos e os estímulos. Os resultados mostraram que a precisão e a sensibilidade foram influenciadas pelo movimento e, em menor grau, pela visão. Em geral, o movimento leva à maior precisão e mais sensibilidade, e a contribuição da visão ocorreu nas condições sem movimento. O nível de confiança não foi influenciado pelo movimento, mas foi influenciado pela visão, sobretudo nas condições sem movimento. O nível de confiança foi congruente à precisão dos julgamentos somente na ausência de visão, sugerindo um conhecimento implícito da precisão.
\end{abstract}

Palavras-chave: sensibilidade cutânea; percepção tátil; percepção somestésica; discriminação de padrão; psicofísica.

\begin{abstract}
Precision, sensitivity and confidence in haptic perception of weight in the presence or absence of movement and vision. In this research we investigated the influence of muscle movement and vision in precision, sensitivity and level of confidence in the haptic perception of weight. The participants compared objects' weight placed on the palms of their hands and, according to the experimental condition, they could or could not make arm movements and make visual contact with the stimuli. The results showed that precision and sensitivity were mainly affected by movement and, to a less extent, by vision. In general, movements produced better precision and sensitivity, and vision contributed only in the conditions without movement. The confidence level was influenced by vision, especially in conditions without movement. Confidence was consistent with precision in conditions without vision, suggesting an implicit knowledge about precision.
\end{abstract}

Keywords: cutaneous sense; tactual perception; somesthetic perception; pattern discrimination; psychophysics.

\section{Resumen}

Precisión, sensibilidad y confiabilidad en la percepción háptica de peso en la presencia o ausencia de movimiento y visión. Este estudio investigó la influencia de los movimientos musculares y visión en la precisión, la sensibilidad y el nivel de confiabilidad en la percepción háptica de peso. Los participantes compararon los pesos colocados en las palmas de las manos y, de acuerdo a la condición experimental, se podían o no mover sus brazos y visualizar o no los estímulos. Los resultados mostraron que la precisión y la sensibilidad han sido influenciados por el movimiento y, en menor medida, por la visión. En general, el movimiento conduce a una mayor precisión y sensibilidad, y la contribución de la visión ocurrió en las condiciones sin movimientos. El nivel de confiabilidad fue afectado por la visión, especialmente en condiciones sin movimientos. El nivel de confianza era congruente con la precisión de los juicios únicamente en ausencia de la visión, lo que sugiere un conocimiento implícito de la precisión.

Palabras clave: sensibilidad cutánea; percepcion tactil; percepcion somestesica; discriminacion de patrones; psicofísica.

A manipulação de objetos é fundamental em nossa vida diária. Uma das principais funções de nossos membros superiores é manipular objetos, e esta ação envolve tanto aspectos motores quanto perceptuais, detectando informações como temperatura, pressão, vibração, textura, consistência e peso (Gibson, 1966; Lederman \& Klatzky, 
1987, 1997; Voisin, Lamarre, \& Chapman, 2002; William, Basso, Case-Smith, \& Nichols-Larsen, 2006). Essas informações são captadas por receptores térmicos e mecânicos situados na pele e em músculos e tendões, ou seja, receptores cutâneos (táteis) e proprioceptivos (cinestésicos) interagem como um sistema integrado que constituí a percepção háptica (Gibson, 1966; Lederman \& Klatzky, 1999, 2009; Loomis \& Lederman, 1986).

Segundo Gibson (1966), a percepção háptica envolve a extração de informação a partir das propriedades dos objetos (informação extereoceptiva), da orientação de segmentos corporais uns em relação aos outros (informação proprioceptiva) e do próprio corpo e seus segmentos em relação ao ambiente ou aos objetos manuseados (informação exproprioceptiva). Gibson (1966) também apontou a existência de três tipos de toques ou subsistemas na percepção háptica. O toque cutâneo caracteriza-se pela estimulação da pele ou tecidos profundos sem que haja movimento de músculos ou articulações. O toque háptico se diferencia do cutâneo pelo movimento de juntas durante a estimulação e o toque dinâmico complementa o háptico ao envolver atividade muscular.

Em pesquisas sobre a percepção háptica é frequente a comparação entre condições com ou sem movimento de músculos e articulações, avaliando assim os componentes cinestésico e tátil. Quando não há movimento, os participantes analisam, por exemplo, estímulos depositados nas palmas de suas mãos ou em seus dedos, não operando propositadamente sobre o estímulo (i.e., toque cutâneo). Por outro lado, quando o participante analisa hapticamente um objeto, há o envolvimento de músculos e articulações relacionado ao pegar, levantar e explorar ativamente o objeto (i.e., toque háptico-dinâmico). Segundo evidências experimentais, a percepção é mais precisa quanto mais rígidos forem os objetos e quanto maior for o envolvimento de músculos e articulações (Debats, Kingma, Beek, \& Smeets, 2012; Lederman \& Klatzky, 1987, 2009).

O estudo da percepção háptica remonta aos primórdios da psicologia científica. Entre os anos de 1829 e 1834, Ernst Weber realizou diversos experimentos sobre as sensações cutâneas e cinestésicas (cf. Weber, Ross, \& Murray, 1996). Em suas pesquisas, Weber aplicou estímulos em diferentes partes do corpo (e.g., abdômen, lábios, pernas e mãos) e verificou que a discriminação entre dois estímulos, isto é, a percepção de que são diferentes, é uma razão entre a mínima diferença percebida (chamada de diferença apenas perceptível ou limiar diferencial) e o estímulo padrão. Em outras palavras, não se trata de uma diferença absoluta de magnitude, mas sim de uma proporção relativa: estímulos de comparação precisam de menos ou mais intensidade para se diferirem de estímulos de intensidade média ou alta, respectivamente. Cada órgão e modalidade sensorial tem um índice de sensibilidade, uma "assinatura" sensorial característica, e quanto menor for essa razão, maior é a sensibilidade ao estímulo e mais precisa é a percepção, isto é, mais precisos são os julgamentos subjetivos com relação ao valor físico do estímulo.

A razão entre a diferença apenas perceptível e o padrão é a primeira lei da psicofísica, também conhecida como fração de
Weber (simbolizada pela letra ' $k$ '), tendo sido calculada para diferentes estímulos e modalidades sensoriais. Por exemplo, a percepção de peso ativa (com movimentação de músculos e articulações) é muito mais sensível $(k=0,02)$ que a percepção de peso passiva, sem movimento $(k=0,2)$ (Brodie \& Ross, 1984; Teghtsoonian, 1971).

A influência da presença ou ausência de movimentos na percepção de peso é bem estabelecida na literatura. A exploração háptica melhora a precisão no julgamento de pesos, conforme observado por Weber e outros estudos (Brodie \& Ross, 1984, 1985; Jones, 1986). A percepção de peso também é influenciada por diversas características dos objetos, por exemplo, tamanho, volume, densidade e textura (Buckingham \& Goodale, 2010; Buckingham, Cant, \& Goodale, 2009; Charpentier, 1891; Cross \& Rotkin, 1975; Flanagan \& Beltzner, 2000; Flanagan \& Wing, 1997; Jones, 1986; Ross \& Di Lollo, 1970; Rule \& Curtis, 1977), além de fatores cognitivos como expectativa e memória (Buckingham \& Goodale, 2010; Ellis \& Lederman, 1998; Reiner, Hecht, Halevy, \& Furman, 2006).

Em tarefas de percepção de peso podem ocorrer ilusões hápticas, caracterizadas por erros de julgamento em função de características visuais e táteis dos objetos (Ellis \& Lederman, 1993; Jones, 1986; Ross, 1969). Por exemplo, a ilusão de tamanho-peso caracteriza-se pelo julgamento de objetos menores como sendo mais pesados do que objetos maiores, mesmo que tenham pesos idênticos (Charpentier, 1891; Ma$\sin \&$ Crestoni, 1988). Essa ilusão é típica da percepção de peso e ocorre mesmo quando não há contato visual com os estímulos. O estudo de Kawai (2002) mostrou que a percepção háptica de tamanho afeta a discriminação de peso, de maneira que o peso percebido aumenta conforme o tamanho percebido diminui.

Segundo o modelo de percepção de peso proposto por Amazeen e Turvey (1996), o peso percebido através das mãos é determinado pela resistência do objeto às forças rotacionais dos membros, ou tensão de inércia pela terminologia da física. Essa resistência é uma propriedade do estímulo, que deriva de sua massa e da distribuição de sua massa (volume e densidade). Por esse motivo, a percepção de peso e a ilusão de tamanho-peso parecem derivar de propriedades como massa, tamanho e volume, quando na verdade se devem à tensão de inércia do objeto (Amazeen \& Turvey, 1996). Esse modelo, portanto, explica diversos dados de percepção de peso, inclusive a ilusão de tamanho-forma, através de uma única propriedade do estímulo, representando um avanço com relação a outros modelos (Amazeen \& Turvey, 1996).

Em suma, a percepção háptica de peso é influenciada por diversos fatores, seja ao nível das características dos estímulos, seja ao nível da presença ou ausência de movimentos musculares e visualização dos estímulos. Considerando que a psicofísica é a área que investiga as relações entre o mundo físico e o mundo percebido através dos órgãos sensoriais, uma questão a ser investigada é se determinadas condições mais ou menos favoráveis a julgamentos mais precisos refletem na confiança dos indivíduos em seus julgamentos. Em outras palavras, se os participantes tiverem que indicar o quanto confiam no julgamento 
realizado, será que condições com presença ou ausência de movimentos ou visão dos estímulos afetariam o nível de confiança nos julgamentos? Será que o fato de movimentar os braços, ou de poder visualizar os estímulos, faz com que os participantes confiem mais em seus julgamentos?

Assim, esta pesquisa teve por objetivo avaliar a influência do movimento muscular e da visão das mãos e estímulos na percepção háptica de peso no que tange à precisão, sensibilidade e confiança dos participantes. Elaboramos um delineamento experimental com quatro tratamentos, conforme a presença ou ausência de movimento e de visão. Investigamos a influência desses fatores em três índices psicofísicos - o limiar diferencial (LD), o ponto de igualdade subjetiva (PIS) e o $\mathrm{k}$ - e no nível de confiança dos participantes. Em particular, o LD e o PIS fornecem medidas independentes a respeito da discriminação de estímulos (detalhes no tópico Cálculos Psícofísicos, na seção Métodos): o LD é uma estimativa de precisão, pois representa o valor a ser adicionado ou subtraído do padrão para que os estímulos de comparação sejam discriminados. Assim, um LD baixo indica alta precisão dos julgamentos, ao passo que um LD alto indica baixa precisão. Por outro lado, o PIS pode ser considerado uma estimativa de acurácia, pois representa o ponto em que os estímulos de comparação são julgados como sendo iguais ao padrão (ponto de igualdade objetiva). Assim, pode-se dizer que houve maior ou menor acurácia conforme o PIS se aproxima ou se afasta do padrão, respectivamente. Já o valor de $\mathrm{k}$, conforme apresentado anteriormente, é um índice de sensibilidade.

O estímulo padrão foi definido em $100 \mathrm{~g}$ e os índices psicofísicos foram calculados em duas condições experimentais. Em uma condição, os estímulos variaram em intervalos de $5 \mathrm{~g}$, representando 1/20 (ou 5\%) do padrão. Em outra condição, os estímulos variavam em intervalos de $2 \mathrm{~g}$, representando $1 / 50$ (ou 2\%) do padrão. Dessa maneira, manipulamos a dificuldade da discriminação de modo a destacar eventuais efeitos das variáveis investigadas.

Com base na literatura, nossa previsão foi que a variável movimento influenciaria os índices psicofísicos e confiança subjetiva nos julgamentos, havendo maior precisão, acurácia, sensibilidade e nível de confiança com a realização de movimentos do que com os braços e mãos em repouso. Além disso, não esperávamos efeitos significativos da variável visão sobre a acurácia, precisão e sensibilidade, dado que o julgamento de pesos é feito pelo sistema háptico e que a visão não traz informações relevantes. Previmos um efeito significativo da visão sobre o nível de confiança, esperando maior confiança quando há visualização dos estímulos, mesmo se tratando de uma informação irrelevante para a tarefa.

\section{Método}

\section{Participantes}

Participaram um total de 72 voluntários (36 mulheres e 36 homens), estudantes universitários entre 20 e 43 anos de idade (Média $=25,9$ anos, $D P=4,3$ ). Os participantes foram subdivididos aleatoriamente em quatro tratamentos experimentais conforme os níveis presente ' + ' ou ausente '-' das variáveis movimento (Mov) e visão (Vis): +Mov+Vis, +MovVis, -Mov+Vis, -Mov-Vis. Foram alocados 18 indivíduos (nove mulheres e nove homens) para cada tratamento.

\section{Materiais}

Os estímulos foram 24 pesos confeccionados com copos plásticos vedados com tampa, com mesmo formato e tamanho: $0,1 \mathrm{~cm}$ de espessura, $10 \mathrm{~cm}$ de altura, diâmetro inferior de $4,5 \mathrm{~cm}$ e diâmetro superior de $8 \mathrm{~cm}$. Dentro dos copos foram colocados pequenos sacos plásticos contendo areia, fixados com algodão na superfície interna de modo a manter controlado o ponto de equilíbrio. Houve dois tipos de estímulo, conforme o intervalo de variação entre os pesos, que poderia ser de 5 ou 2 gramas: 12 copos pintados com tinta atóxica de cor preta, um estímulo padrão (100 g) e outros 11 copos de 75 a 125 g (intervalos de 5), e 12 copos pintados com tinta atóxica de cor branca, um estímulo padrão e outros 11 copos de 90 a $110 \mathrm{~g}$ (intervalos de 2). Os estímulos de cada tipo foram numerados de 1 a 12 em ordem crescente de peso e era apenas de conhecimento do experimentador.

Neste experimento também utilizamos duas cadeiras (uma para o participante e outra para o experimentador), uma mesa de metal na cor branca, com superfície de 90 x $90 \mathrm{~cm}$ e $70 \mathrm{~cm}$ de altura, e uma estrutura metálica que servia de suporte para uma cortina confeccionada em tecido denso na cor preta, na qual dois pequenos orifícios permitiam a passagem dos braços, mantendo as mãos fora do campo visual do participante.

\section{Procedimento}

Os participantes foram testados em sessões únicas e individuais, iniciadas com a leitura e assinatura do termo de consentimento, informações sobre as regras do experimento e instruções sobre a tarefa. Durante a sessão, os participantes não portavam relógios, pulseiras, anéis e telefone celular.

Os estímulos foram apresentados aos pares: primeiramente o estímulo padrão era anunciado e colocado sobre a palma da mão direita (ou esquerda) do participante e, em seguida, o estímulo de comparação era anunciado e colocado sobre a palma da mão esquerda (ou direita) do participante, permanecendo o par de estímulos por um período de 30 s. Então, o participante deveria informar se o estímulo de comparação era "mais pesado", "menos pesado" ou "igual" em relação ao estímulo padrão. Em seguida, deveria informar seu nível de confiança no julgamento realizado, sinalizando em uma escala de 1 (sem confiança) a 7 (absoluta confiança), semelhante à utilizada em estudo sobre percepção de comprimento (Osaka, 1987).

Utilizamos o método psicofísico dos estímulos constantes. Em uma sequência de provas, os 11 estímulos de comparação foram apresentados de maneira aleatória, sempre mantendo a mão sobre a qual eram apoiados. Ao término de uma sequência de provas, outra sequência se iniciava, invertendo a mão 
sobre a qual foram apoiados o estímulo padrão e os estímulos de comparação. Uma sequência foi realizada como treinamento, seguida por 14 sequências de 11 provas experimentais, ou seja, 154 provas para cada tipo de variação de estímulo (intervalos de 2 e $5 \mathrm{~g}$ ), totalizando 308 provas válidas. A planilha de registro de respostas consistia de duas tabelas (uma para cada tipo de estímulo), com 14 linhas representando a sequência de provas e 11 colunas representando o peso que foi comparado ao padrão. O experimentador anotava a resposta do participante na coluna correspondente ao estímulo de comparação apresentado, usando os seguintes códigos: '-' para "menos pesado", ‘=' para "peso igual” e '+' para "mais pesado”, junto ao respectivo valor de confiança no julgamento.

Em nosso delineamento experimental, distribuímos os participantes em quatro condições de acordo com os fatores investigados, a saber, a presença ou ausência de movimento muscular e de visão dos estímulos. Nos tratamentos envolvendo movimento muscular (+Mov), os participantes foram instruídos a movimentar os antebraços durante a comparação dos estímulos; já nos tratamentos sem movimento muscular (-Mov), os participantes foram instruídos a não realizar qualquer tipo de movimento durante a comparação dos estímulos, mantendo os antebraços apoiados sobre a mesa com as palmas das mãos voltadas para cima. Nos tratamentos em que não havia visão dos estímulos (-Vis), uma cortina densa e escura impedia que os participantes visualizassem suas mãos e os estímulos sobre elas. Nos tratamentos com visão dos estímulos (+Vis), nada impedia a visão das mãos e dos estímulos sobre elas.

\section{Cálculos psicofísicos}

A partir do protocolo de respostas do participante, primeiramente somamos o número de ocorrência de cada tipo de resposta ('-', '=' e '+') para cada um dos 11 estímulos de comparação em cada tabela. Em seguida, aplicamos o procedimento de "redução": Cada resposta incongruente (por exemplo, responder "mais pesado" para um estímulo de comparação de $95 \mathrm{~g}$ ) cancela a ocorrência de uma resposta congruente para aquele estímulo, e adiciona-se o número de itens cancelados (neste caso '2', um '+' e outro '-') à contagem de itens '=' (i.e., itens de não-discriminação) (cf. da Silva \& Rozestraten, s.d., p. 61).

Feita a redução, calculamos a taxa de respostas de discriminação (D) e não-discriminação $(\mathrm{nD})$ para cada estímulo de comparação, ou seja, D/14 e nD/14. Esses valores foram então normalizados, isto é, transformados em z-scores e representados em dois gráficos (um para discriminação '-' e outro para '+') com a magnitude dos estímulos no eixo x (nota-se que o valor de 0,5 tem $z=0$ ). Finalmente, em cada gráfico foi calculada a reta de regressão linear dos pontos, sendo que o ponto da reta de regressão que cruza o eixo y $=0$ (isto é, $z=0$ ) representa uma estimativa do ponto em que o estímulo é reconhecido 50\% das vezes (cf. Gescheider, 1997, pp. 45-54). Dessa maneira, obtivemos o ponto limiar inferior (PLI) baseado nas discriminações '-' e o ponto limiar superior (PLS) baseado nas discriminações '+'. A partir desses dois valores limiares, é possível extrair todos os índices psicofísicos de interesse.
Utilizamos como variáveis dependentes o PIS, dado pela média dos pontos limiares (PLI + PLS)/2, o limiar diferencial (LD), dado pela metade da diferença entre os pontos limiares (PLS - PLI)/2 , a fração de Weber (k) dada por LD/PIS e o nível de confiança nos julgamentos congruentes, isto é, que foram realizados corretamente. Cumpre notar que, na prática, a fração de Weber não é calculada com o valor do estímulo padrão (igualdade objetiva), mas sim com o valor de PIS, pois este representa como o estímulo padrão é percebido pelo participante (da Silva \& Rozestraten, s.d.).

\section{Resultados}

\section{Análises preliminares}

Em vista de equívocos cometidos no protocolo de registro de respostas de 13 participantes, tivemos que descartar esses dados. Trabalhamos, portanto, com uma amostra de 59 participantes.

Primeiramente, analisamos os dados de cada variável dependente através do teste de Shapiro-Wilk para testar a hipótese nula (com nível de significância $\mathbf{U}=0,05$ ) de que os dados são provenientes de uma distribuição normal. Em todos os casos, o teste não permitiu descartar a hipótese de normalidade, seja para o PIS com $5 \mathrm{~g}(p=0,38)$ e $2 \mathrm{~g}(p=0,075)$, seja para o LD com $5 \mathrm{~g}(p=0,99)$ e $2 \mathrm{~g}(p=0,29)$, seja para o $\mathrm{k} \operatorname{com} 5 \mathrm{~g}(p$ $=0,99)$ e $2 \mathrm{~g}(p=0,26)$, seja para a confiança com $5 \mathrm{~g}(p=0,23)$ e $2 \mathrm{~g}(p=0,22)$. Em vista disso, optamos pelo uso de análises estatísticas paramétricas, exceto no caso do PIS com 2 g, cuja distribuição apresentou uma assimetria à direita e consideramos adequado empregar um teste não-paramétrico.

\section{Análises estatísticas}

Analisamos separadamente os dados de cada variável dependente (PIS, LD, k e confiança) e intervalo de estímulo (5 g e $2 \mathrm{~g}$ ) por meio de análises de variância (ANOVA) para avaliar os efeitos do movimento muscular e visão. Utilizamos o nível de significância $\alpha=0,05$, o $\eta_{p}^{2}$ como medida de tamanho de efeito e, em caso de interação significativa, o teste post-hoc com correção de Bonferroni. No caso do PIS com 2 g, utilizamos a análise de variância não-paramétrica (teste de Kruskal-Wallis).

A Figura 1 apresenta os dados referentes ao PIS. Para os estímulos de $5 \mathrm{~g}$, observamos um efeito significativo do movimento $\left[F_{(1,55)}=4,27, p=0,044, \eta_{p}^{2}=0,072\right]$, pois houve maior acurácia com +Mov (PIS = 98,8) do que com -Mov (PIS = 97,7). Houve também um efeito significativo da visão $\left[F_{(1,55)}=7,29\right.$, $\left.p=0,009, \eta_{p}^{2}=0,117\right]$, dada a maior acurácia com +Vis (PIS $=99,1)$ do que com -VIS (PIS = 97,5). A interação entre movimento e visão não foi significativa $\left[p=0,97, \eta_{p}^{2}<00\right]$. Com relação aos estímulos de $2 \mathrm{~g}$, pode-se observar na Figura 1 que as médias são relativamente próximas, com intercruzamento das barras de erro padrão, e de fato não houve efeito significativo de movimento ou visão [ $p=0,59]$. 


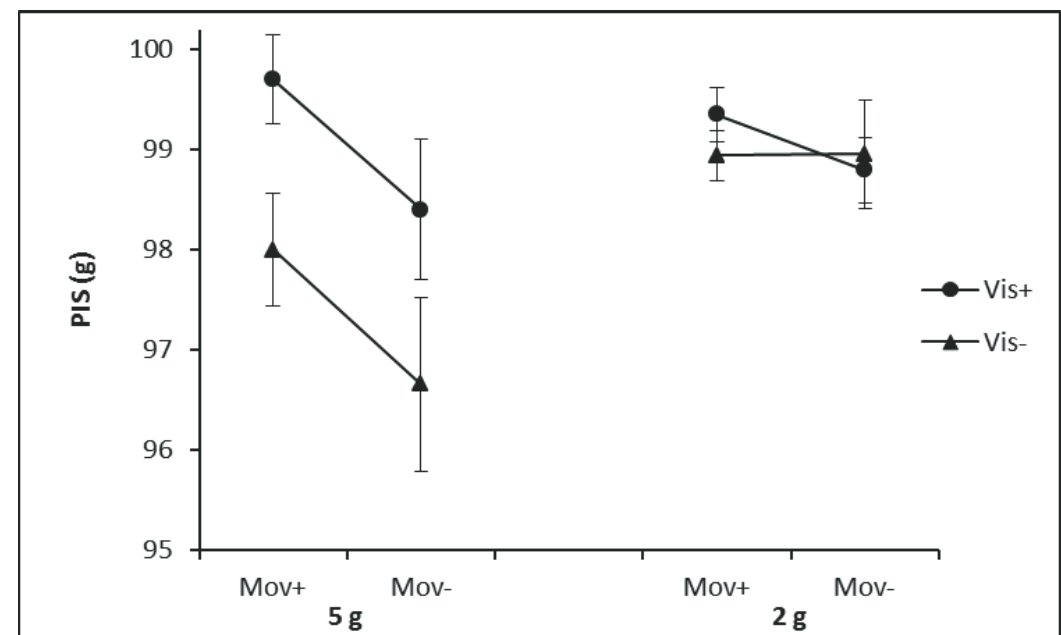

Figura 1. Valores Médios do Ponto de Igualdade Subjetiva (PIS) de Acordo com a Presença (+) ou Ausência (-) de Movimento (Mov) e Visão (Vis). As Barras de Erro Representam o Erro Padrão da Média

A Figura 2 apresenta os dados referentes ao LD. Para os estímulos de $5 \mathrm{~g}$, observamos um efeito significativo do movimento $\left[F_{(1,55)}=27,2, p<0,001, \eta_{p}^{2}=0,33\right]$, pois houve maior precisão (ou seja, menor valor de $\mathrm{LD}$ ) com $+\operatorname{Mov}(\mathrm{LD}=7,82$ ) do que com -Mov $(\mathrm{LD}=11,26)$. Não houve efeito significativo da visão $\left[p=0,45, \eta_{p}^{2}=0,01\right]$ ou interação entre movimento e visão $\left[p=0,27, \eta_{p}^{2}=0,02\right]$. Com relação aos estímulos de 2 $\mathrm{g}$, houve um efeito significativo do movimento $\left[F_{(1,55)}=28,3\right.$, $\left.p<0,001, \eta_{p}^{2}=0,34\right]$, visto a maior precisão com $+\operatorname{Mov}$ (LD =
$5,04)$ do que com -Mov (LD = 6,72). Houve um efeito significativo da visão $\left[F_{(1,55)}=8,66, p=0,005, \eta_{p}^{2}=0,136\right]$, pois houve maior precisão com + Vis $(\mathrm{LD}=5,42)$ do que com -Vis $(\mathrm{LD}=$ $6,12)$. A interação entre movimento e visão não foi significativa, $\left[p=0,13, \eta_{p}^{2}=0,04\right]$, e apresentou um efeito fraco ( $4 \%$ da variância, como se pode notar pelo valor de $\eta_{p}^{2}$ ) em vista do efeito principal da visão ter sido proveniente de -Mov, conforme se pode notar na Figura 2.

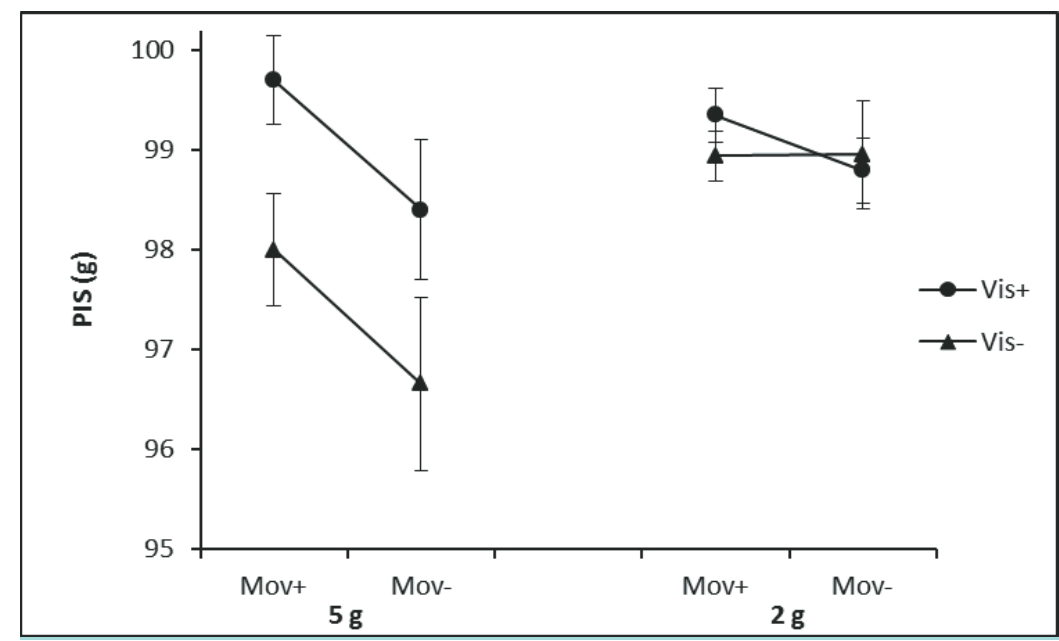

Figura 2. Valores Médios do Limiar Diferencial (LD) de Acordo com a Presença (+) ou Ausência (-) de Movimento (Mov) e Visão (Vis). As Barras de Erro Representam o Erro Padrão da Média

A Figura 3 mostra os dados referentes ao k. Para os estímulos de $5 \mathrm{~g}$, houve um efeito significativo do movimento $\left[F_{(1,}\right.$ 55) $\left.=29,16, p<0,001, \eta_{p}^{2}=0,347\right]$, visto a maior sensibilidade (menor valor de $\mathrm{k})$ com $+\operatorname{Mov}(\mathrm{k}=0,079)$ do que com $-\mathrm{Mov}(\mathrm{k}$ $=0,115)$. Não houve efeito significativo de visão $\left[p=0,33, \eta^{2}\right.$ $=0,02]$, ou interação significativa entre movimento e visão [ $p$ $\left.=0,29, \eta_{p}^{2}=0,02\right]$. Com relação aos estímulos de $2 \mathrm{~g}$, houve efeito significativo do movimento $\left[F_{(1,55)}=27,78, p<0,001, \eta^{2}\right.$ $=0,336]$, dada a maior sensibilidade com $+\operatorname{Mov}(k=0,051)$ do que com -Mov $(\mathrm{k}=0,068)$. Houve efeito significativo da visão $\left[F_{(1,55)}=8,47, p=0,005, \eta_{p}^{2}=0,133\right]$, com maior sensibilidade com + Vis $(k=0,055)$ do que com -Vis $(k=0,062)$. A interação entre movimento e visão não foi significativa $\left[p=0,14, \eta^{2}=\right.$ $0,039]$. Similar ao ocorrido com o LD, nota-se na Figura 3 pouca diferença de $\mathrm{k}$ com + Vis e -Vis na presença de movimento (+Mov), e um pequeno efeito da visão na ausência de movimento (-Mov), caracterizada por uma queda na sensibilidade na condição-Mov-Vis. 


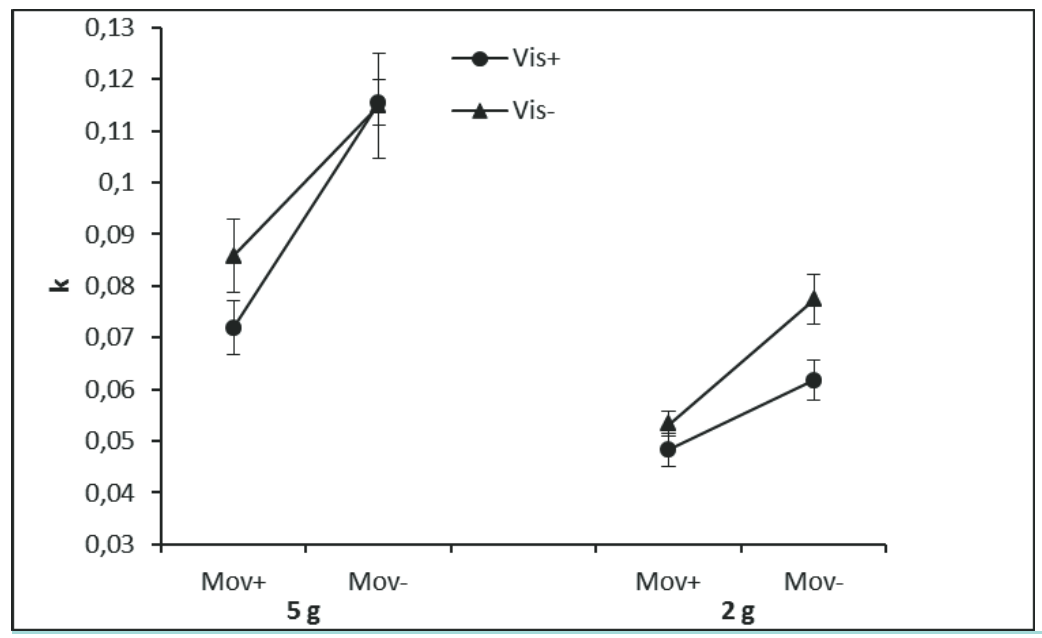

Figura 3. Valores Médios da Fração de Weber $(k)$ de Acordo com a presença $(+)$ ou Ausência (-) de Movimento (Mov) e Visão (Vis). As Barras de Erro Representam o Erro Padrão da Média

Por fim, a Figura 4 apresenta os dados referentes à confiança. Para os estímulos de $5 \mathrm{~g}$, não observamos efeito do movimento $\left[p=0,67, \eta_{p}^{2}<00\right]$, e houve um efeito significativo da visão $\left[F_{(1,55)}=6,44, p=0,014, \eta_{p}^{2}=0,105\right]$, dada a diferença entre + Vis (confiança $=5,37)$ e -Vis (confiança $=5,12)$. A interação foi significativa $\left[F_{(1,55)}=4,9, p=0,03, \eta_{p}^{2}=0,082\right]$, pois com + Mov não houve diferença entre +Vis e -Vis $(5,23$ e 5,19, respecti- vamente) $[p=1]$, mas para -Mov houve diferença entre +Vis $(5,53)$ e -Vis $(4,98)$ [ $p=0,018]$. Com relação aos estímulos de 2 $\mathrm{g}$, não houve efeito do movimento $\left[p=0,56, \eta^{2}<00\right]$, e houve um efeito significativo da visão $\left[F_{(1,55)}=10,2, p=0,002, \eta^{2}=\right.$ $0,156]$, dada a diferença entre + Vis (confiança $=5,29)$ e - Vis (confiança $=4,94)$. A interação não foi significativa $[p=0,15$, $\left.\eta_{p}^{2}=0,037\right]$, mas seguiu o mesmo padrão observado com 5 g.



Figura 4. Valores Médios do Nível de Confiança de Acordo com a Presença (+) ou Ausência (-) de Movimento (Mov) e Visão (Vis). As Barras de Erro Representam o Erro Padrão da Média

\section{Discussão}

Primeiramente, cumpre fazer uma breve retomada sobre os índices psicofísicos escolhidos e suas interpretações. Conforme mencionamos, o ponto de igualdade subjetiva (PIS) e o limiar diferencial (LD) são duas medidas independentes sobre a discriminação de estímulos. Ambas são obtidas a partir dos pontos limiares superior e inferior, mas através de operações distintas. O PIS é dado pela média dos pontos limiares e é uma estimativa do ponto em que os estímulos de comparação são julgados como sendo iguais ao padrão, representando como o padrão é percebido subjetivamente. O PIS pode ser interpre- tado como acurácia visto que indica a direção de aproximação ou afastamento subjetivo do padrão, isto é, do ponto de igualdade objetiva. Já o LD é a metade da diferença entre o limiar superior e inferior, representando uma estimativa de qual valor deve ser adicionado ou subtraído do padrão para que um estímulo de comparação seja discriminado. Em vista disso, o LD pode ser interpretado como precisão da discriminação. Cumpre notar que o LD relaciona-se com o conceito de sensibilidade, por exemplo, um LD baixo indica capacidade de discriminar estímulos mais próximos ao padrão e, portanto, maior sensibilidade. De fato, a fração de Weber (k) é dada pela razão LD/ PIS, ou seja, a relação entre a magnitude da mínima diferença percebida e a magnitude subjetiva do padrão. 
Selecionamos os dados de dois participantes (+Mov+Vis, com 5 g) para exemplificar os conceitos de acurácia, precisão e sensibilidade, assim como a independência entre PIS e LD e a relação entre LD e k. O participante 2 , cujo limiar inferior foi 87,2 e superior foi 109,3, teve PIS = 98,3, LD = 11,1 e $\mathrm{k}=0,11$. Por outro lado, o participante 15, cujo limiar inferior foi 91,0 e superior foi 105,7 , teve PIS $=98,4, \mathrm{LD}=7,3$ e $\mathrm{k}=0,07$. Notase que esses participantes apresentaram níveis semelhantes de acurácia, embora tenham diferido em precisão e sensibilidade.

Feitas essas considerações, podemos discutir os resultados de nossa pesquisa, cujo objetivo foi avaliar a influência da presença ou ausência de movimento e visão na acurácia, precisão, sensibilidade e nível de confiança da discriminação háptica de peso. Em geral, os resultados mostraram que movimento e visão influenciam as variáveis estudadas, confirmando algumas de nossas previsões e contrariando outras. A seguir, discutimos os efeitos observados sobre os índices psicofísicos e, posteriormente, discutimos os resultados sobre o nível de confiança.

A presença ou ausência de movimento influenciou a acurácia, precisão e sensibilidade, conforme havíamos previsto. Efeitos fortes e consistentes foram observados principalmente sobre a precisão e a sensibilidade, visto que explicam em torno de $33 \%$ (i.e., valores de $\eta_{p}^{2}$ entre 0,33 e 0,34) da variância de LD e $\mathrm{k}$ nas duas condições de estímulos investigadas (5 g e 2 g). Em específico, observamos maior precisão e sensibilidade (i.e., menores valores de $\mathrm{LD}$ e $\mathrm{k}$, respectivamente) com +Mov em comparação a -Mov (cf. Figuras 2 e 3). Esses resultados são congruentes aos relatados pela literatura - a discriminação de pesos melhora significativamente com o envolvimento do componente háptico (+Mov) em comparação ao componente tátil (-Mov) (Amazeen \& Turvey, 1996; Brodie \& Ross, 1984, 1985; Jones, 1986)

Com relação à acurácia, contudo, o efeito do movimento foi fraco $\left(p=0,044, \eta_{p}^{2}=0,072\right)$ e somente ocorreu com variações de $5 \mathrm{~g}$, condição em que também houve efeito da visão ( $p$ $=0,009, \eta_{p}^{2}=0,117$ ). Considerando o padrão observado na Figura 1 e a ausência de interação, movimento e visão exerceram efeitos independentes e aditivos sobre os valores de PIS: tanto + Mov quanto +Vis favoreceram um valor de PIS mais próximo ao padrão, sendo que o maior afastamento foi observado em -Mov-Vis. Na condição que exigiu descriminações mais finas (2 g), não houve efeitos significativos do movimento ou da visão. Trata-se de um resultado curioso, visto que essa condição deveria acentuar os efeitos das variáveis investigadas, como de fato foi observado com LD e k. Em vista disso e dos efeitos relativamente fracos observados com $5 \mathrm{~g}$, nossa interpretação é que o PIS foi menos susceptível a influências dos fatores investigados. A percepção subjetiva do padrão não é um índice robusto para informar sobre a qualidade das discriminações, conforme podemos depreender do exemplo referente aos participantes 2 e 15 .

A possibilidade de visualizar ou não as mãos e os estímulos influenciou a precisão e a sensibilidade, ao contrário do que havíamos previsto. Contudo, os efeitos observados foram menos robustos do que os observados com movimento. A visão não exerceu efeito sobre as discriminações com variações de $5 \mathrm{~g}$, e exerceu efeitos moderados sobre o LD e k com variações de 2 g, explicando cerca de $13 \%$ da variância (i.e., valores de $\eta_{p}^{2}$ de
0,13). O efeito da visão teve uma interação fraca e não-significativa com movimento (cerca de $4 \%$ da variância, ou valores de $\eta_{p}^{2}$ em torno de 0,04). Como pode ser observado nas Figuras 2 e 3 (variações $2 \mathrm{~g}$ ), com + Mov as médias e as barras de erro padrão de LD e k foram próximas entre si e sobrepostas com relação a +Vis ou -Vis. Contudo, em -Mov observa-se um afastamento das médias e das barras de erro padrão, com maior precisão e sensibilidade na presença de visão (+Vis) do que na ausência de visão (-Vis).

Esses resultados sugerem uma melhora da precisão e sensibilidade com a visualização das mãos e estímulos, sobretudo quando a discriminação depende do componente tátil (i.e., sem movimento) do sistema háptico. Tal resultado vai ao encontro da literatura que aponta a integração entre os sistemas háptico e visual (Ernst \& Banks, 2002; Taylor-Clarke, Kennett, \& Haggard, 2002). De fato, diversos estudos mostraram que olhar as mãos melhora a acuidade tátil das mãos, mesmo que a visão não seja informativa sobre as características do estímulo avaliadas, como é o caso do presente estudo (Kennett, TaylorClarke, \& Haggard, 2001; Taylor-Clarke et al., 2002; Tipper et al., 1998). De maneira geral, a visão melhora a percepção háptica (Newport, Rabb, \& Jackson, 2002) e evidências neurofisiológicas mostram que a visão modula o processamento cortical somatosensorial (Taylor-Clarke et al., 2002).

Os resultados acima discutidos indicam que foi importante testar dois intervalos de variação dos estímulos de comparação, visto que os efeitos variaram de acordo com o grau de discriminação exigido. A discriminação mais fina exigida na condição 2 g (1/50 do padrão) fez emergir o efeito da visão sobre a precisão e sensibilidade, o que não foi possível detectar na condição $5 \mathrm{~g}$ (1/20 do padrão). Além disso, foi possível identificar que o efeito da visão incide sobretudo sobre o componente tátil.

Por fim, resta-nos discutir como o nível de confiança foi influenciado pelos fatores investigados. As análises mostraram que não houve efeito principal do movimento sobre a confiança, contrariando nossa previsão inicial. Ou seja, mesmo havendo um efeito robusto do movimento sobre os índices de precisão e sensibilidade, o fato de poder movimentar ou não os braços não influenciou o nível de confiança dos participantes em seus julgamentos. Trata-se de um resultado curioso, visto que seria plausível esperar que menores níveis de confiança fossem observados na situação em que não havia movimentação dos braços. Em nossa vida diária, quando somos confrontados com uma tarefa de estimar o peso de algum objeto ou comparar pesos entre objetos, intuitivamente movimentamos os braços para avaliá-los. Apesar de não exercer um efeito principal sobre a confiança, a variável movimento interagiu com a visão, conforme será discutido abaixo.

A visão influenciou significativamente o nível de confiança, de maneira que maiores índices ocorreram nas condições em que era possível visualizar as mãos e os estímulos. Esse resultado era esperado, dado que a visão é mais um canal sensorial a fornecer informações, mesmo que irrelevantes, elevando o nível de confiança dos participantes. Também houve uma interação significativa entre visão e movimento: nas condições com movimento, que levou a julgamentos mais precisos e maior sensibilidade, não houve diferença entre os níveis de confiança com ou sem visão. Entretanto, na ausência de movimento, 
houve uma discrepância entre as condições com e sem visão, de maneira que menores níveis foram obervados em -Mov-Vis do que em -Mov+Vis. Ou seja, a visão afetou a confiança nos julgamentos feitos pelo componente tátil (-Mov), mas não pelo componente háptico (+Mov).

Resta-nos verificar até que ponto a confiança foi congruente com a qualidade das discriminações. Para tal, comparamos a oscilação da confiança (Figura 4) com a oscilação da acurácia (Figura 1, variações 5 g) e da precisão (Figura 2, variações 5 g e 2 g) conforme + Vis e -Vis. A confiança foi congruente com a acurácia somente com -Vis, isto é, observa-se uma queda na acurácia e na confiança em -Mov-Vis. Por outro lado, a confiança foi incongruente com a acurácia para + Vis, de modo que se observa uma queda na acurácia e aumento de confiança de + Mov+Vis para -Mov+Vis. Esses padrões se mantiveram no caso do LD: precisão e confiança caíram para -Mov-Vis, ao passo que a precisão caiu e a confiança aumentou em -Mov+Vis. Tais resultados indicam que a possibilidade de visualizar as mãos e estímulos confunde a confiança subjetiva nas discriminações, levando aos participantes a superestimarem seus julgamentos. $\mathrm{Na}$ ausência de visão, os participantes fazem uma apreciação mais adequada de seus julgamentos, sugerindo um certo conhecimento implícito sobre a acurácia e precisão das discriminações.

Em conjunto, nossos resultados trouxeram informações relevantes sobre a percepção háptica de peso. Precisão e sensibilidade foram influenciadas principalmente pelo movimento e, em menor grau, pela visão. Embora sejam resultados semelhantes aos já descritos pela literatura, a presente pesquisa avançou ao manipular movimento e visão no mesmo experimento, investigando seus efeitos principais (e interações) sobre os índices psicofísicos. Em particular, é interessante notar como os componentes tátil e háptico da discriminação de pesos podem ser diferencialmente afetados - em nosso estudo, pela visão, mas pesquisas futuras poderão explorar diferenças entre julgamentos táteis e hápticos de outras variáveis (por exemplo, tamanho, volume, densidade e textura).

\section{Referências}

Amazeen, E. L., \& Turvey, M. T. (1996). Weight perception and the haptic sizeweight illusion are functions of the inertia tensor. Journal of Experimental Psychology: Human Perception and Performance, 22(1), 213-232. doi: 10.1037/0096-1523.22.1.213

Brodie, E. E., \& Ross, H. E. (1984). Sensorimotor mechanisms in weight discrimination. Perception \& Psychophysics, 36(5), 477-481. doi: 10.3758/ BF03207502

Brodie, E. E., \& Ross, H. E. (1985). Jiggling a lifted weight does aid discrimination. American Journal of Psychology, 98(3), 469-471. doi: 10.2307/1422630

Buckingham, G., \& Goodale, M. A. (2010). Lifting without seeing: The role of vision in perceiving and acting upon the size weight illusion. PLoS One, 5(3), e9709. doi: 10.1371/journal.pone.0009709

Buckingham, G., Cant, J. S., \& Goodale, M. A. (2009). Living in a material world: How visual cues to material properties affect the way that we lift objects and perceive their weight. Journal of Neurophysiology, 102(6), 3111-3118. doi: 10.1152/jn.00515.2009

Charpentier, A. (1891). Analyse experimentale de quelques elements de la sensation de poids. Archives de Physiologie Normale et Pathologiques, 3, 122 135.

Cross, D. V., \& Rotkin, L. (1975). The relation between size and apparent heaviness. Perception \& Psychophysics, 18(2), 79-87. doi: 10.3758/ BF03204091
Da Silva, J. A., \& Rozestraten, R. J. A. (s.d.). Manual Prático de Psicofísica. Recuperado de pge3.wikispaces.com/file/view/manual+de+psicofisica.PDF

Debats, N. B., Kingma, I., Beek, P. J., \& Smeets, J. B. (2012). Moving the Weber fraction: The perceptual precision for moment of inertia increases with exploration force. PloS One, 7(9), e42941. doi: 10.1371/journal. pone.0042941

Ellis, R. R., \& Lederman, S. J. (1993). The role of haptic versus visual volume cues in the size-weight illusion. Perception \& Psychophysics, 53(3), 315-324. doi: 10.3758/BF03205186

Ellis, R. R., \& Lederman, S. J. (1998). The golf-ball illusion: Evidence for topdown processing in weight perception. Perception, 27(2), 193-201. doi: $10.1068 / \mathrm{p} 270193$

Ernst, M. O., \& Banks, M. S. (2002). Humans integrate visual and haptic information in a statistically optimal fashion. Nature, 415(6870), 429-433. doi: 10.1038/415429a

Flanagan, J. R., \& Beltzner, M. A. (2000). Independence of perceptual and sensorimotor predictions in the size-weight illusions. Nature Neuroscience, 3(7), 737-741. doi: 10.1038/76701

Flanagan, J. R., \& Wing, A. M. (1997). Effects of surface texture and grip force on the discrimination of hand-held loads. Perception and Psychophysics, 59(1), 111-118. doi: 10.3758/BF03206853

Gescheider, G. A. (1997). Psychophysics: The fundamentals. Mahwah: Lawrence Erlbaum Associates.

Gibson, J. J. (1966). The senses considered as perceptual systems. Oxford: Houghton Mifflin.

Jones, L. A. (1986). Perception of force and weight: Theory and research Psychological Bulletin, 100(1), 29-42. doi: 10.1037/0033-2909.100.1.29

Kawai, S. (2002). Heaviness perception. I. Constant involvement of haptically perceived size in weight discrimination. Experimental Brain Research, 147(1), 16-22. doi: 10.1007/s00221-002-1209-3

Kennett, S., Taylor-Clarke, M., \& Haggard, P. (2001). Noninformative vision improves the spatial resolution of touch in humans. Current Biology, 11(15), 1188-1191. doi: 10.1016/S0960-9822(01)00327-X

Lederman, S. J.; \& Klatzky, R. L. (1987). Hand movements: A window into haptic object recognition. Cognitive Psychology, 19(3), 342-368. doi:10.1016/00100285(87)90008-9

Lederman, S. J., \& Klatzky, R. L. (1997). Haptic aspects of motor control. In F. Boller \& J. Grafman (Orgs.), Handbook of neuropsychology (Volume 11, pp. 131-148). Amsterdam: Elsevier Science Publishers.

Lederman, S. J., \& Klatzky, R. L. (1999). Haptic perception: A tutorial. Attention Perception, \& Psychophysics, 71(7), 1439-1459. doi: 10.3758/APP.71.7.1439

Lederman, S. J., \& Klatzky, R. L. (2009). Haptic perception: A tutorial. Attention, Perception, \& Psychophysics, 71(7), 1439-1459. doi: 10.3758/APP.71.7.1439

Loomis, J. M., \& Lederman, S. J. (1986). Tactual perception. In Boff, K., Kaufman, L., \& Thomas, J. (Orgs.), Handbook of perception and human performance (Volume 2, Cap. 31, pp. 31-1, 31-41). Nova Iorque: Wiley.

Masin, S. C., \& Crestoni, L. (1988). Experimental demonstration of the sensory basis of the size-weight illusion. Perception \& Psychophysics, 44(4), 309-312. doi: 10.3758/BF03210411

Newport, R., Rabb, B., \& Jackson, S. R. (2002). Noninformative vision improves haptic spatial perception. Current Biology, 12(19), 1661-1664. doi: 10.1016/ S0960-9822(02)01178-8

Osaka, N. (1987). Psicofisica mental para la longitud percibida, el area y los mapas geograficos: Una aproximacion psicofisica a la representacion de la memoria visual. Revista Latinoamericana de Psicologia, 19(3), 337-352. Recuperado de http://www.redalyc.org/pdf/805/80519306.pdf

Reiner, M., Hecht, D., Halevy, G., \& Furman, M. (2006). Semantic interference and facilitation in haptic perception. In EuroHaptics Society (Org.), Proceedings of the Eurohaptics 2006 (pp. 31-35). Paris.

Ross, H. E. (1969). When is a weight not illusory? The Quarterly journal of experimental psychology, 21(4), 346-355. doi:10.1080/14640746908400230

Ross, J., \& Di Lollo, V. (1970). Differences in heaviness in relation to density and weight. Perception \& Psychophysics, 7(3), 161-162. doi: 10.3758/ BF03208648 
276 Percepção háptica de peso

Rule, S. J., \& Curtis, D. W. (1977). The influence of the interaction of weight and volume on subjective heaviness. Perception \& Psychophysics, 22(2), 159-164. doi: 10.3758/BF03198749

Taylor-Clarke, M., Kennett, S., \& Haggard, P. (2002). Vision modulates somatosensory cortical processing. Current Biology, 12(3), 233-236. doi: 10.1016/S0960-9822(01)00681-9

Teghtsoonian, R. (1971). On the expoents in Stevens' law and the constant in Ekman's law. Psychological Review, 78(1), 71-80. doi: 10.1037/h0030300

Tipper, S. P., Lloyd, D., Shorland, B., Dancer, C., Howard, L. A., \& McGlone, F. (1998). Vision influences tactile perception without proprioceptive orienting. Neuroreport, 9(8), 1741-1744. doi: 10.1097/00001756199806010-00013

Voisin, J., Lamarre, Y., \& Chapman, C. E. (2002). Haptic discrimination of object shape in humans: Contribution of cutaneous and proprioceptive inputs. Experimental Brain Research, 145(2), 251-260. doi: 10.1007/s00221-002$1118-5$

Weber, E. H., Ross, H. E., \& Murray, D. J. (1996). E. H. Weber on the tactile senses. Hove: Erlbaum (UK) Taylor \& Francis.

Williams, P. S., Basso, D. M., Case-Smith, J., \& Nichols-Larsen, D. S. (2006) Development of the Hand Active Sensation Test: Reliability and validity. Archives of Physical Medicine and Rehabilitation, 87(11), 1471-1477. doi: 10.1016/j.apmr.2006.08.019 
Márcio Rogério Penha, Doutor em Ciências na área de Psicobiologia pela Faculdade de Filosofia, Ciências e Letras de Ribeirão Preto, Universidade de São Paulo (FFCLRP-USP), é Técnico de Laboratório no setor de Citogenética Humana da Faculdade de Medicina de Ribeirão Preto (FMRP-USP). Endereço para correspondência: Departamento de Genética, Bloco D, FMRP. Avenida dos Bandeirantes, 3900, Ribeirão Preto (SP). CEP 14049-900. Telefone: (16) 3315-3141. E-mail: mrpenha2000@yahoo.com.br

Ricardo Basso Garcia, Doutor em Ciências na área de Psicobiologia pela Faculdade de Filosofia, Ciências e Letras de Ribeirão Preto, Universidade de São Paulo (FFCLRP-USP), é Pós-doutorando no Instituto de Psicologia da Universidade Federal de Uberlândia (UFU). E-mail: rbgarcia@gmail.com

Igor Otto Douchkin, é Técnico de Laboratório na Faculdade de Filosofia, Ciências e Letras de Ribeirão Preto, Universidade de São Paulo (FFCLRP-USP). E-mail: douchkin@ffclrp.usp.br

José Aparecido da Silva, Pós-doutor em Percepção e Psicofísica pela Universidade da Califórnia, é Professor Titular do Departamento de Psicologia da Faculdade de Filosofia, Ciências e Letras de Ribeirão Preto, Universidade de São Paulo (FFCLRP-USP). E-mail: jadsilva@ffclrp.usp.br 